\title{
Alternating Wavelet-Time Finite Element Method: Modeling and Analysis of Nonlinear Wave Propagation in One and Two-Dimensional Waveguides
}

\author{
Yu Liu, Andrew J. Dick* \\ Nonlinear Phenomena Laboratory \\ Department of Mechanical Engineering and Materials Science \\ Rice University \\ Houston, Texas
}

\begin{abstract}
In this paper, a novel method named the alternating wavelet-time finite element method (AWT-FEM) is proposed for studying elastic wave propagation in nonlinear structures. An alternating iterative procedure between the time-domain and a wavelet-domain combined with the spectral finite element method (SFEM) is employed to solve wave equations with general nonlinearities. The advantages of the proposed method are (1) the potential to provide high fidelity results for impacts with high frequency content through the use of the spectral finite element method; (2) nonlinear structures with physically realistic boundary conditions can easily be studied by circumventing wrap-around issues associated with Fourier-based methods; (3) the parallel computing compatible framework and the semi-analytical nature of SFEM make it more computationally efficient for nonlinear systems modeled with structural components. Simulations using the proposed method are conducted to demonstrate its applicability to study nonlinear wave propagation in one-dimensional and two-dimensional systems.
\end{abstract}

Keywords: Nonlinear Wave Propagation, Wavelets, Finite Element Method

\section{Introduction}

In the vast majority of traditional scientific and technological applications, wave propagation in solid structures is bounded by the elastic limit and a linear approximation is often adopted. However, as the exploration is entering into more advanced fields like new materials, extreme working environments, and high fidelity analysis, nonlinearity can be introduced in various ways and can become a crucial factor that must not be neglected. In the past decade, facilitated by the development of numerical techniques and high performance computers, significant progress has been made in the study of nonlinear elastic wave propagation for applications including biomedical analysis [1], non-destructive evaluation [2], and seismic motion analysis [3].

In many military and civil applications, nonlinear mechanical wave propagation occurs through structural components that can be approximated as rods, beams, or plates. For example, in the force identification of aircraft through impact wave analysis, the impact load often has a high amplitude with a short duration, where high frequency content in the response will significantly strengthen the influence of the nonlinear properties [4]. The wings of the aircraft can be approximated as a beam or plate structure with a geometric nonlinearity. Another example is in the monitoring and control of drill strings in the oilfield. Drill strings can be modeled as long geometrically nonlinear beams with various cross sections and material properties [5]. Elastic waves caused by impacts with the wellbore and stress waves in the mud flow can be used to control

\footnotetext{
*Address all correspondence to this author

Email addresses: liu.yu@rice.edu (Yu Liu), Andrew.J.Dick@rice.edu (Andrew J. Dick)

Preprint submitted to Journal of Sound and Vibration
}

November 26, 2015 
the stability [6]. A third example is in the dynamics of high-speed and high-precision parallel robots [7]. Each link of the robot can be modeled as a beam undergoing large deformation. For these systems and many other, exact solutions of linear wave equations for rod, beam, and plate structures are known [8] and analytical relationships in the nonlinear wave equation are also well established [9]. Incorporating those results with a general numerical framework will greatly improve the computational performance and provide significantly improved accuracy.

Based on the specific nature of nonlinear elastic wave propagation, a good numerical technique should fulfill three requirements. (1) Provide the ability to predict high fidelity responses - Nonlinear phenomenon can be accurately captured in the response and available for further analysis. (2) Provide high computational efficiency - The method should take advantage of current computation technology to ensure studying larger models is not computational prohibitive. (3) Be flexible - The method should be able to be applied to various structures for a range of working constraints. With the above considerations, a new numerical method named the alternating wavelet-time finite element method (AWT-FEM) is proposed to study nonlinear elastic wave propagation in one-dimensional (1D) and two-dimensional (2D) structures.

\subsection{Literature Review}

A comparison of existing methods is shown in Table 1. There are five examination criteria: (1) demonstrated or potential for high fidelity performance, (2) applicability to study linear behavior, (3) applicability to study nonlinear behavior, (4) ability to represent physically realistic boundary constraints, and (5) parallel computing compatibility. The check mark $(\checkmark)$ indicates that the criterion is satisfied, the dash mark $(-)$ indicates the criterion is partially satisfied, and the $\mathrm{X}$ mark $(\times)$ indicates that the criterion is not satisfied. The AWT-FEM, which satisfies all criteria, is introduced in this paper.

Table 1: Comparison of different methods.

\begin{tabular}{cccccc}
\hline \multicolumn{7}{c}{ Table 1: Comparison of different methods. } \\
\hline Methods & High Fidelity & Linear & Nonlinear & Boundary & Parallel \\
\hline TFEM [10] & $\times$ & $\checkmark$ & $\checkmark$ & $\checkmark$ & - \\
MTFEM [11] & $\checkmark$ & $\checkmark$ & $\checkmark$ & $\checkmark$ & - \\
SFEM [12] & $\checkmark$ & $\checkmark$ & $\times$ & - & $\checkmark$ \\
WSFEM [13] & $\checkmark$ & $\checkmark$ & $\times$ & $\checkmark$ & $\checkmark$ \\
AFT-FEM [14] & $\checkmark$ & $\checkmark$ & $\checkmark$ & - & $\checkmark$ \\
AWT-FEM & $\checkmark$ & $\checkmark$ & $\checkmark$ & $\checkmark$ & $\checkmark$ \\
\hline
\end{tabular}

The finite element method (FEM) [10] has been widely applied to study structural dynamics and has developed into a class of numerical methods with various time integration and space discretization techniques. Here, the time-domain FEM (TFEM) refers to a standardized procedure composed of a serial timeintegration technique like the Newmark method, an iteration approach like the Newton-Raphson method to address nonlinearity, and a standard finite element space discretization to approximate the geometry. Other modified versions of FEM with various time-integration techniques like the space-time finite element method are not considered here [35]. The TFEM can be used to solve both linear and nonlinear problems with physically realistic boundary conditions and complex geometries. However, for transient wave propagation, especially those caused by impact loading with high frequency content, responses predicted with this method can exhibit spurious oscillations which results from erroneously introduced numerical dispersion and dissipation $[11,15]$. This error will accumulate as the wave travels a long distance and will significantly distort the response unless an extremely fine meshing is adopted to capture the sharp gradient change in the wave shape. Also, due to the serial nature of the Newmark method, TFEM cannot be directly incorporated into a parallel computing framework. Procedures to improve computational efficiency are needed to make it compatible with a parallel computational framework. Other parts of the method may also be able to benefit from parallel processing.

In order to solve the spurious oscillation error and obtain a high fidelity response, many modified timedomain finite element methods (MTFEM) have been developed by using more advanced interpolation functions $[11,16]$. The spectral element method (SEM), first proposed by Patera [17] in fluid dynamics, has 
been adapted to study 1D linear wave propagation in solid structures [16]. In SEM, high order polynomials have been chosen as local shape functions and a high degree of accuracy in the response can be observed. An application of SEM to study 1D nonlinear wave propagation in a rod was reported in [18]. Even though theoretically SEM can be adapted to solve nonlinear problems, other related works are surprisingly rare in the literature. Ham and Bathe proposed another modified method using low-order polynomials enriched with harmonic functions [11]. A specific scheme has also been designed to overcome ill-conditioning. This method can be used to solve both linear and materially nonlinear systems, but may encounter challenges for geometrically nonlinear systems. Additional efforts to reformulate SEM are needed to make these methods compatible for parallel computing framework.

Another class of methods transforms the model and solution procedure into a spectral domain. A typical example is the fast Fourier transform (FFT) based spectral finite element method (SFEM) proposed by Doyle $[8,12]$. In the literature, there is some confusion over the terms SFEM and SEM [16]. Here, SFEM specifically refers to the Fourier-based method developed by Doyle. For linear problems, SFEM uses exact wave solution as frequency-dependent shape functions and only one element is required to obtain highly accurate responses for a given position in a structural component like a rod or a beam. For a structure like a frame or a truss, an element-based procedure can be adopted at each independent frequency. This makes the method inherently suitable for parallel computing. With the incorporation of the exact analytical wave solutions, the capacity for parallel computing and the fast computational performance of FFT, the SFEM can be utilized as a real-time method to obtain high fidelity responses to impacts with high frequency content [4]. Various applications on linear 1D and 2D problems can be found in Gopalakrishnan and Lee's works $[19,20]$. However, this method has two major drawbacks. First, the periodic nature of the basis functions used for the Fourier transform can result in wrap-around issues in which the signal outside the time-window will become wrapped around to the beginning of the signal sequence. Semi-infinite elements (non-reflecting boundary condition) are often added to the model at the ends of a structure to leak energy out of the system. For other types of boundary conditions, additional damping combined with a longer time-window is required. This greatly deteriorates the computational performance of SFEM when it is applied to study wave propagation in structures with physically realistic boundary constraints. Second, nonlinear terms often result in convolutions in the frequency-domain, which are computationally prohibitive to calculate with iterative procedures. This makes SFEM not directly applicable for studying nonlinear systems.

In order to avoid the wrap-around issue of SFEM, Mitra and Gopalakrishnan developed a wavelet-based spectral finite element method (WSFEM) [13]. Instead of using FFT, a discrete wavelet transform (DWT) on the sampling scale was adopted to transform the linear wave equation into a wavelet-domain where each wavelet point represents a shifting along the time axis. A wavelet extrapolation technique was also incorporated to represent non-periodic boundary conditions [21]. WSFEM successfully circumvented the wrap-around issue and can also be incorporated into a parallel computing framework after being uncoupled through an eigenvalue analysis. Applications to linear wave propagation in composite and nano-composite structures were reported in [22]. An adaptation of the wavelet transformation technique in WSFEM with SEM spatial interpolation was also applied to 2D and 3D linear elastic waves [23, 24]. Similar to SFEM, WSFEM cannot be directly applied to study nonlinear systems.

An intuitive solution to deal with the nonlinear problem in frequency-domain methods is to transform the calculation of the nonlinear terms back to the time-domain. An alternating frequency-time iterative framework is needed to transform the result of the nonlinear calculation into the frequency-domain, solve for the linear solution by using SFEM, and then return to the time-domain to calculate new values of the nonlinear terms for the next iteration. This framework was first proposed by Cameron [25]. A combination of it with the SFEM was performed by Lee [26] to study 1D nonlinear blood flow in human arteries. Based on that, the authors of this paper developed an alternating frequency-time finite element method (AFT-FEM) to study nonlinear elastic wave propagation [14, 31]. AFT-FEM has proven to be more computationally efficient than TFEM with the combined advantages of parallel computing and the analytical nature of SFEM. In [31], the high fidelity of the AFT-FEM is systematically examined through comparison with TFEM for extreme impact loading conditions. As shown by the study of the numerical errors of TFEM, through decreasing the impact duration and therefore increasing the frequency content of the signal, the 
TFEM requires an increasing number of elements to capture the sharp gradient change in the response and eventually fails to produce an accurate response when the impact duration is decreased to $T_{p}=20 \mu$ s even with 100 elements for a 1D rod model. A comparison of convergence between AFT-FEM and TFEM in [31] also showed that the accuracy of AFT-FEM is at least an order of magnitude better than TFEM with the same number of elements. However, since FFT is adopted as the transform technique, its application is limited for structures with physically realistic boundary conditions and often requires semi-infinite boundary elements (non-reflecting boundary conditions) so that the wrap-around issue can be avoided. For other types of boundary conditions, additional damping combined with an extended time window is required and may result in convergence issues.

In this paper, a new numerical technique named the alternating wavelet-time finite element method (AWT-FEM) is developed to study nonlinear elastic wave propagation. The method adapts a wavelet Galerkin's method [27] as the transform technique, uses an alternating iterative procedure between the wavelet-domain and the time-domain to address the nonlinearity, uses exact linear wave solutions as interpolation functions, and uses a finite element framework to represent the structure's geometry. It can produce accurate responses and be applicable to linear and nonlinear systems with physically realistic boundary conditions. Its computational performance is significantly improved through the use of parallel computing.

\subsection{Background}

A brief introduction of wavelet transformation is presented in this subsection. The discrete wavelet transform (DWT), with the orthogonal compactly supported wavelet family developed by Daubechies [32], is adopted as the foundation for the development of the single-scale wavelet Galerkin's method [27] used in this study.

At a given scale $i$, the basis function or scaling function $\psi_{i, k}$ is obtained from the mother scaling function $\psi(t)$ with the following equation:

$$
\varphi_{i, k}=2^{i / 2} \varphi\left(2^{i} t-k\right), \quad k \in \mathbb{Z}
$$

where $i$ and $k$ are the dilation and translation indices. By increasing the value of $i$, the wavelets become more compact and the level of details increases. The index $i$ can also be viewed as the scale, and $k$ is the translation of the wavelet at a given scale $i$. The index $k$ represents a series of points shifting along the time axis. The values of the scaling function $\varphi(t)$ are calculated iteratively by using the cascade algorithm [32] on a fine grid of points on $t$. The wavelet function $\psi(t)$ can be calculated from $\varphi(t)$. Since only the scaling function $\varphi(t)$ will be used in this study, the generation of $\psi(t)$ and the discussion of wavelet functions are omitted here.

Various wavelet families have been proposed by researchers to perform analysis of signals. Daubechies developed a set of compactly supported wavelets known as the Daubechies wavelets [32], which are employed in this study. They are a family of specially designed wavelets that have orthogonal scaling functions and a maximum number of vanishing moments. They cannot be expressed in closed form and are normally computed by using the cascade algorithm. For specific details regarding properties and expressions of Daubechies wavelets, refer to [32]. The theory is originally designed to perform a multi-resolution analysis by presenting the transformed information in time/space and scale/frequency domains. Coefficients for the Daubechies wavelets were obtained by using the 'dbwavf' command in MATLAB for this study. Although implementation of discrete wavelets is performed by using the two sets of coefficients, the concepts are presented here making use of analog representations for ease of understanding. In this study, the scaling function $\varphi_{i, k}$ at one given scale (i.e. the finest scale) is adopted as the basis/shape function for the Galerkin's method. The scale is determined by the input signal. For example, the input data has $N$ points. The finest scale is a dilation $i$ that can represent all the sampled points.

Some important properties of $\varphi(t)$ related to the applications in this study are listed as follows:

(1) Normalization

The area under the scaling function is considered to be unity.

$$
\int_{-\infty}^{\infty} \varphi(t) d t=1
$$


(2) Orthogonality

For Daubechies wavelets, the integer of a scaling functions and a translated version of itself are orthogonal $[22,32]$ :

$$
\int_{-\infty}^{\infty} \varphi(t+l) \varphi(t+k) d t=\delta_{l, k}, \quad k \in \mathbb{Z}
$$

where $\delta_{l, k}=1$ when $l=k$, otherwise $\delta_{l, k}=0$.

\subsection{Outline}

The remainder of this paper is organized in the following manner. The AWT-FEM is developed in the second section. The applications of AWT-FEM on a rod, a beam, and a plate model are derived in this section. In the third section, the results of numerical simulations are presented. First, 1D axial wave propagation in a materially nonlinear rod caused by impact loading is studied with AWT-FEM, AFT-FEM, and TFEM. Comparisons of the results are performed and the computational performance of these three methods is examined to observe the advantages of AWT-FEM. Second, 1D transverse wave propagation in a geometrically nonlinear beam is studied by using AWT-FEM. The interactions between the nonlinear wave propagation and physically realistic boundary conditions are also investigated. Finally, 2D transverse wave propagation in a plate with a weak geometric nonlinearity is studied with the AWT-FEM to demonstrate its applicability to higher dimensional structures. Concluding remarks and the direction of future work are provided in the fourth section.

\section{Alternating Wavelet-Time Finite Element Method}

A specific wavelet transform is described in the first subsection. The spectral finite element method formulation in the wavelet-domain is discussed in the second section. The procedure of AWT-FEM is described in the third subsection. Its applications to an elementary rod model with a material nonlinearity, an Euler-Bernoulli beam model with a geometric nonlinearity, and a conventional plate model with a weak geometric nonlinearity are derived in the last subsection.

\subsection{Spectrally-Uncoupled Wavelet Transform}

The general governing differential wave equation of a nonlinear structure is given as [26]

$$
\mathcal{L} \mathbf{u}(\mathbf{x}, t)+\mathcal{M} \ddot{\mathbf{u}}(\mathbf{x}, t)+\mathcal{N} \dot{\mathbf{u}}(\mathbf{x}, t)+\mathbf{f}(\mathbf{u}, \mathbf{x}, t)=\mathbf{p}(\mathbf{x}, t),
$$

where $\mathcal{L}$ is the linear differential operator with respect to the spatial coordinate vector $\mathbf{x}$, the over-dot represents a derivative with respect to time $t, \mathcal{M}$ is the inertial operator, $\mathcal{N}$ is the damping operator, $\mathbf{u}(\mathbf{x}, t)$ is the displacement field vector, $\mathbf{p}(\mathbf{x}, t)$ is the external load vector, and $\mathbf{f}(\mathbf{x}, t)$ is the nonlinear term vector.

A single-scale wavelet Galerkin's method using a Daubechies wavelet is applied to transform Eqn. (4) into a set of spectrally-uncoupled partial differential equations (PDEs). Details of this method and its application to a 1D linear wave equation can be found in [13, 21, 27]. Here, the application of this method to the general nonlinear wave equation in Eqn. (4) is briefly described in the following three steps.

\section{Step 1:}

A new variable $\tau=t / \Delta t$ is introduced. In the following derivation, $\tau$ is regarded as a continuous variable since integration with respect to $\tau$ will be performed. The variable $\Delta t$ corresponds to the time step of the signal.

The function $\overline{\mathbf{u}}(\mathbf{x}, \tau)$, which is equivalent to $\mathbf{u}(\mathbf{x}, t)$ for the new time variable $\tau$, is represented in an expansion form by translating the wavelet scaling function $\varphi(\tau)$.

$$
\overline{\mathbf{u}}(\mathbf{x}, \tau)=\sum_{k} \mathbf{u}_{k}(\mathbf{x}) \varphi(\tau-k), \quad k \in \mathbf{Z}
$$


where $\mathbf{u}_{k}(\mathbf{x})$ (referred to as $\mathbf{u}_{k}$ hereafter) is the approximation coefficient of $\mathbf{u}(\mathbf{x}, t)$ at an associated point $k$ and $\mathbf{Z}$ is the set of integers. By utilizing the orthogonality of the scaling function as defined in Eqn. (3), the approximation coefficient $\mathbf{u}_{k}$ is obtained as

$$
\mathbf{u}_{k}=\Delta t \int_{-\infty}^{\infty} \overline{\mathbf{u}}(\mathbf{x}, \tau) \varphi(\tau-k) d \tau .
$$

The scaling function $\varphi(t)$ is defined on the finest scale (the sampling scale). Hence, the integer $k$ presents a shifting along the sampled time axis for $k$ sample points. It is comparable to the frequency component at a given frequency in the Fourier expansion in the sense that both the expansion in Eqn. (5) and Fourier expansion expand the original function by a class of basis function indexed by a variable. The physical meaning of $k$ indicates that the expansion in Eqn. (5) is essentially a shifting tool for a time integration technique. The index $k$ is referred as the "wavelet point" to distinguish it from the time series index $\tau$ before the expansion. Similarly, the nonlinear term $\mathbf{f}(\mathbf{x}, t)$ and the loading term $\mathbf{p}(\mathbf{x}, t)$ are expanded by $\mathbf{f}_{k}$ and $\mathbf{p}_{k}$ as follows.

$$
\begin{aligned}
\mathbf{f}(\mathbf{x}, t) & =\sum_{k} \mathbf{f}_{k}(x) \varphi(\tau-k), \quad k \in \mathbf{Z}, \\
\mathbf{p}(\mathbf{x}, t) & =\sum_{k} \mathbf{p}_{k}(x) \varphi(\tau-k), \quad k \in \mathbf{Z},
\end{aligned}
$$

where $\mathbf{f}_{k}(x)$ and $\mathbf{p}_{k}(x)$ are abbreviated as $\mathbf{f}_{k}$ and $\mathbf{p}_{k}$ here after.

The above expansions are substituted into Eqn. (4). By multiplying the resulting equation by the scaling function $\varphi(\tau-j)$ and integrating it over time, the following set of equations are obtained.

$$
\begin{aligned}
& \mathcal{L} \mathbf{u}_{j}+\int_{-\infty}^{\infty} \frac{\mathcal{M}}{\Delta t^{2}} \sum_{k} \mathbf{u}_{k} \ddot{\varphi}(\tau-k) \varphi(\tau-j) d \tau \\
& +\int_{-\infty}^{\infty} \frac{\mathcal{N}}{\Delta t} \sum_{k} \mathbf{u}_{k} \dot{\varphi}(\tau-k) \varphi(\tau-j) d \tau+\mathbf{f}_{j}=\mathbf{p}_{j}, j=0,1, \ldots, n-1,
\end{aligned}
$$

where $\mathbf{f}(\mathbf{x}, t), \mathbf{p}(\mathbf{x}, t)$, and the $\mathbf{u}(\mathbf{x}, t)$ after the linear differential operator $\mathcal{L}$ are transformed into their corresponding approximation coefficients $\mathbf{f}_{j}, \mathbf{p}_{j}$, and $\mathbf{u}_{j}$ due to the orthogonality of the wavelet scaling function, as shown in section 1.2. For the time derivatives of $\mathbf{\mathbf { u }}(\mathbf{x}, \tau)$, the two integrals associated with $\mathcal{M}$ and $\mathcal{N}$ in Eqn. (9) are evaluated in the form of connection coefficients for compactly supported wavelets [27], as defined in Eqn. (10) and Eqn. (11).

$$
\begin{aligned}
& \Omega_{j-k}^{1}=\int_{-\infty}^{\infty} \dot{\varphi}(\tau-k) \varphi(\tau-j) d \tau, \\
& \Omega_{j-k}^{2}=\int_{-\infty}^{\infty} \ddot{\varphi}(\tau-k) \varphi(\tau-j) d \tau,
\end{aligned}
$$

where the superscript 1 and 2 refer to on which order of derivative of $\Omega$ the operation is performed and should not be confused with exponents. Details about the evaluation of the connection coefficients are provided through an example of a rod model in the first section of the appendix. A matrix form of Eqn. (9) for a 1D model is also provided. The index $j$ represents a given wavelet point. Similar to the definition of $k$, it indicates a shifting along the time axis.

For a multidimensional problem, $\mathbf{u}=[u, v, w]^{T}$ and $\mathbf{u}_{j}=\left[u_{j}, v_{j}, w_{j}\right]^{T}$, a complete matrix form of Eqn. (9) with respect to the three dimensions and the wavelet point $j$ is cumbersome. The couplings between dimensions $(x, y, z)$ are assumed to be represented in the nonlinear term $\mathbf{f}_{\mathbf{j}}$. Since $\mathbf{f}_{\mathbf{j}}$ will be treated as an equivalent nodal force term without explicitly evaluating it and only its nodal values will be used in the numerical procedure to solve the equation, Eqn. (9) can be regarded as decoupled with respect to dimensions. The coupling in Eqn. (9) is with respect to the index $j$ and is captured in the matrix $\boldsymbol{\Omega}$. Hence, 
Eqn. (9) describes a set of spectrally coupled PDEs with respect to $j$, and has to be solved for each wavelet point $j$, which corresponds to a translation along time axis.

\section{Step 2:}

Next, a wavelet extrapolation technique [21] is adopted to address the time boundaries. Specific treatments of periodic and non-periodic time boundaries for linear wave equations can be found in [13]. This method uses a polynomial of order $N / 2-1$ to extrapolate the values at the boundaries from the known coefficients near the boundaries. An example of the construction of the non-periodic boundary for a 1D rod model is provided in the second section of the appendix. The connection coefficient matrix $\Omega$ becomes a $n \times n$ matrix $\boldsymbol{\Lambda}$, which is a version with non-periodic time boundaries. The result of this process is arranged into three decoupled matrix forms with respect to the three dimensions, each of which will be further decoupled with respect to the spectral index $j$ in the next step.

\section{Step 3:}

A standard eigenvalue analysis is performed to uncouple the matrix form of Eqn. (9) with respect to $j$. An example of this decoupling process for the $x$-dimension is provided in the third subsection of appendix. The eigenvalues and the $n \times n$ eigenvector matrix of the $n \times n$ connection coefficient matrix $\Lambda$ are $\lambda_{j}, j=0,1, \cdots, n-1$ and $\Phi$, respectively. The decoupling process is independent of dimensions and can be implemented with respect to each spatial dimension separately. After each dimension is spectrally uncoupled, the result can be organized as

$$
\mathcal{L} \hat{\mathbf{u}}_{j}+\left(\mathcal{M} \lambda_{j}^{2}+\mathcal{N} \lambda_{j}\right) \hat{\mathbf{u}}_{j}+\hat{\mathbf{f}}_{j}=\hat{\mathbf{p}}_{j}, \quad j=0,1, \ldots, n-1,
$$

where the eigenvalue $\lambda_{j}$ can be treated as a pseudo-frequency and is related to the physical angular frequency $\omega_{j}$ in a Fourier transform through $\lambda_{j}=i \omega_{j}$, the hat symbol ' ${ }^{\prime \prime}$ indicates the decoupled version and

$$
\begin{aligned}
\hat{\mathbf{u}}_{j} & =\left[\hat{u}_{j}, \hat{v}_{j}, \hat{w}_{j}\right]^{T}, \\
\hat{\mathbf{f}}_{j} & =\left[\hat{F}_{u j}, \hat{F}_{v j}, \hat{F}_{w j}\right]^{T}, \\
\hat{\mathbf{p}}_{j} & =\left[\hat{P}_{u j}, \hat{P}_{v j}, \hat{P}_{w j}\right]^{T}, \\
\left\{\hat{u}_{j}\right\} & =\Phi^{-1}\left\{u_{j}\right\}, \\
\left\{\hat{v}_{j}\right\} & =\Phi^{-1}\left\{v_{j}\right\}, \\
\left\{\hat{w}_{j}\right\} & =\Phi^{-1}\left\{w_{j}\right\}, \\
\left\{\hat{F}_{u j}\right\} & =\Phi^{-1}\left\{F_{u j}\right\}, \\
\left\{\hat{F}_{v j}\right\} & =\Phi^{-1}\left\{F_{v j}\right\}, \\
\left\{\hat{F}_{w j}\right\} & =\Phi^{-1}\left\{F_{w j}\right\}, \\
\left\{\hat{P}_{u j}\right\} & =\Phi^{-1}\left\{P_{u j}\right\}, \\
\left\{\hat{P}_{v j}\right\} & =\Phi^{-1}\left\{P_{v j}\right\}, \\
\left\{\hat{P}_{w j}\right\} & =\Phi^{-1}\left\{P_{w j}\right\},
\end{aligned}
$$

where the bold lower case indicates that the variable is a vector, the brackets '[ ]' indicate a conventional three dimensional spatial vector, and the accolades ' \{\} ' indicate a vector formation with respect to $j$.

These three steps define a special single-scale wavelet transform to reduce a temporal problem into a set of spectrally-uncoupled PDEs. This procedure involves several wavelet-based numerical techniques [21, 27], an eigenvalue analysis, and an inverse calculation of matrices. However, it is worth noting that this procedure is independent of the system to be studied and must only be performed once for a given wavelet scaling 
function $\varphi(t)$ and length of the time window $n$. The resulting eigenvalues $\lambda_{j}$, eigenvector matrix $\Phi$, and the inverse of $\Phi$ can then be calculated and stored for future use. As defined in Section 1.2, the scaling function is defined at the sampling rate of the signal, which is also the finest scale achievable for a given set of data. For example, for an input data with $N$ points, the sampling rate is $1 / N$. In the above wavelet transform procedure, the approximation coefficients of a given signal are first obtained by using a numerical version of Eqn. (6). The vector of approximation coefficients, which has the same length as the original signal, is multiplied by the inverse of the eigenvector matrix $\Phi$ in order to obtain the corresponding values in the defined wavelet-domain. The inverse transform is then performed by multiplication by the eigenvector matrix $\Phi$ to obtain the approximation coefficients in the time-domain. The signal is finally recovered by using Eqn. (5).

\subsection{Spectral Finite Element Method Formulation}

Various methods are available to solve the linear version of Eqn. (12). In [23, 24], SEM is chosen to solve general linear 2D and 3D problems in the wavelet-domain. In this study, the research focus is to develop a numerical technique for structures that can be modeled with rod, beam, and plate elements, like the applications mentioned in the introduction section. Utilizing the analytical wave solutions of those models may greatly improve the computational efficiency and alleviate potential convergence issues with the iterative approach that will be constructed to address nonlinearities.

The linear homogeneous wave equation is obtained by removing the nonlinear term and external force term in Eqn. (12).

$$
\mathcal{L} \hat{\mathbf{u}}_{j}+\left(\mathcal{M} \lambda_{j}^{2}+\mathcal{N} \lambda_{j}\right) \hat{\mathbf{u}}_{j}=0, \quad j=0,1, \ldots, n-1 .
$$

For 1D problems, $\mathbf{x}=x$ and Eqn. (12) is a set of nonlinear ODEs. Exact solutions to Eqn. (14) for rod and beam structures are well-established [8]. In linear SFEM, those exact solutions are directly employed to solve for the response at a given location $x$ and only one element is needed for the formulation. In this nonlinear derivation, the exact linear solutions are adopted as the shape functions in a standard finite element framework in the wavelet-domain. The spectral displacement vector $\hat{\mathbf{u}}_{j}$ is expressed as

$$
\hat{\mathbf{u}}_{j}=\mathbf{N}\left(x ; \lambda_{j}\right) \mathbf{q}_{j},
$$

where $\mathbf{N}\left(x ; \lambda_{j}\right)$ (referred as $\mathbf{N}$ hereafter) is the spectral shape functions based on the exact linear wave solutions, and $\mathbf{q}_{j}$ is the spectral nodal displacement vector at the pseudo-frequency $\lambda_{j}$. By following a standard Galerkin's method, the nonlinear ODE in Eqn. (12) becomes the following matrix form equation

$$
\mathbf{K}\left(\lambda_{j}\right) \mathbf{q}_{j}=\mathbf{q}_{\mathbf{E}}+\mathbf{q}_{\mathbf{N}},
$$

where

$$
\begin{aligned}
\mathbf{K}\left(\lambda_{j}\right) & =\int_{0}^{L_{e}}\left(\mathbf{A}(\mathbf{N}, \mathbf{N})+\mathbf{N}^{T}\left(\mathcal{M} \lambda_{j}^{2}+\mathcal{N} \lambda_{j}\right) \mathbf{N}\right) d x \\
\mathbf{q}_{\mathbf{E}} & =\int_{0}^{L_{e}} \mathbf{N}^{T} \mathbf{p}_{j} d x \\
\mathbf{q}_{\mathbf{N}} & =\int_{0}^{L_{e}} \mathbf{N}^{T} \mathbf{f}_{j} d x,
\end{aligned}
$$

and $L_{e}$ is the length of the element.

The spectrally formulated dynamic stiffness matrix is represented by $\mathbf{K}\left(\lambda_{j}\right)$ and $\mathbf{A}(\mathbf{N}, \mathbf{N})$ is the function resulting from integration by parts [20]. The function $\mathbf{A}(\mathbf{N}, \mathbf{N})$ represents a general operation and is expressed for three cases below in Eqn. (23), Eqn. (31), Eqn. (32), and Eqn. (39). The term $\mathbf{q}_{\mathbf{E}}$ is the equivalent nodal force term for the distributive external load, and $\mathbf{q}_{\mathbf{N}}$ is the equivalent nodal force term for the nonlinear term. It is worth noting that the process to derive Eqn. (16) may involve several steps of integration by parts, so the sign on the right-hand side may change depending on the explicit form of $\mathcal{L}$. The expression in Eqn. (16) is for the purpose of a general and concise representation. 
For a 2D problem, $\mathbf{x}=[x, y]$ and Eqn. (12) is a set of nonlinear PDEs. The wavelet transform defined in Section 2.1 is performed again on Eqn. (12) with respect to one of the spatial coordinate $y$. After that, Eqn. (12) is reduced to a set of ODEs and the model is simplified into a 1D problem. A similar spectral finite element method formulation as above can be performed to obtain a matrix form as in Eqn. (16). An example of this spatial reduction process for a plate model is provided at the end of this section.

\subsection{General Procedure of the AWT-FEM}

Based on the spectrally-uncoupled wavelet transform and the spectral finite element method formulation in the wavelet-domain, an alternating wavelet-time framework is constructed to iteratively simulate nonlinear elastic wave propagation responses. A flowchart of the AWT-FEM is presented in Fig. 1. The initial state is obtained from the linear model by setting the nonlinear term $\mathbf{f}(\mathbf{u}, \mathbf{x}, t)$ in Eqn. (4) equal to zero. The nonlinear term is rewritten as $\mathbf{f}(\mathbf{u}, \mathbf{x}, t)$ to indicate that the value of the nonlinear force term $\mathbf{f}$ is directly determined by the current value of $\mathbf{u}$, which it is a function of position $\mathbf{x}$ and time $t$. At each iteration, the nodal values of the nonlinear term $\mathbf{f}(\mathbf{u}, \mathbf{x}, t)$ are calculated from the current states in the time-domain. The resulting response and the nodal values of external force $\mathbf{p}(\mathbf{x}, t)$ are transformed into the wavelet-domain through the spectrally-uncoupled wavelet transform. At each pseudo-frequency, a spectral finite element method formulation is constructed based on Eqn. (16) to obtain nodal values of $\hat{\mathbf{u}}_{j}$. Nodal values of the derivatives of $\hat{\mathbf{u}}_{j}$ with respect to $\mathbf{x}$ are calculated based on Eqn. (15). Since Eqn. (12) is uncoupled with respect to $j$, the above calculation is performed in parallel at each wavelet point. The nodal information is then transformed back to the time-domain via the inverse wavelet transform. New values of the nonlinear term $\mathbf{f}(\mathbf{x}, t)$ are calculated for use in the next iteration. The process is continued until the error measure defined in Eqn. (18) is less than a prescribed tolerance.

The number of wavelet point is determined by the length of the signal. The higher the sampling rate and the longer the signal length, the better accuracy of the wavelet transform and a better performance of the AWT-FEM since Eqn. (5) and Eqn. (6) are implemented through numerical integration in the program. In most of the applications, the signal is collected through experiments or predefined as inputs. Hence, the length of the signal or the number of wavelet point is taken as fixed conditions in numerical simulation in this study.

$$
\beta_{i}=\frac{1}{M} \sum_{m=1}^{M}\left\|\frac{\mathbf{f}_{i}\left(\mathbf{x}_{0}, t_{m}\right)-\mathbf{f}_{i-1}\left(\mathbf{x}_{0}, t_{m}\right)}{\mathbf{f}_{i}\left(\mathbf{x}_{0}, t_{m}\right)}\right\|,
$$

where $M$ is the number of sampling points, $\mathbf{x}_{0}$ is the location of the excitation, and the subscript $i$ represents the iteration number. The response at the impacted position has both the initial impulse profile and the interaction with the reflected wave from the boundary within a given time-window. It can reflect most of the accumulated nonlinear information in the wave propagation process. Hence, the impact position is chosen to define the error measure. Other points could also be used and the result will not be qualitatively different.

\subsection{Applications to Structural Models}

(1) Rod:

A rod model with a material nonlinearity is considered here. A general nonlinear stress-strain relationship with a high order strain term has been used in $[9,14,18,29]$. In the second order case, the nonlinear constitutive law becomes

$$
\sigma=E\left(\epsilon+\alpha \epsilon^{2}\right),
$$

where $\sigma$ is the stress, $\epsilon$ is the strain, $E$ is the elastic modulus, and $\alpha$ is the nonlinear coefficient. When $\alpha>0$, the quadratic term exhibits a hardening effect on the constitutive curve. When $\alpha<0$, a softening effect can be observed.

A linear strain-displacement relationship $\epsilon=d u / d x$ is assumed. By using Hamilton's principle, the governing materially nonlinear homogeneous wave equation for a rod is obtained as

$$
E A u^{\prime \prime}-\rho A \ddot{u}+F(x, t)=0,
$$




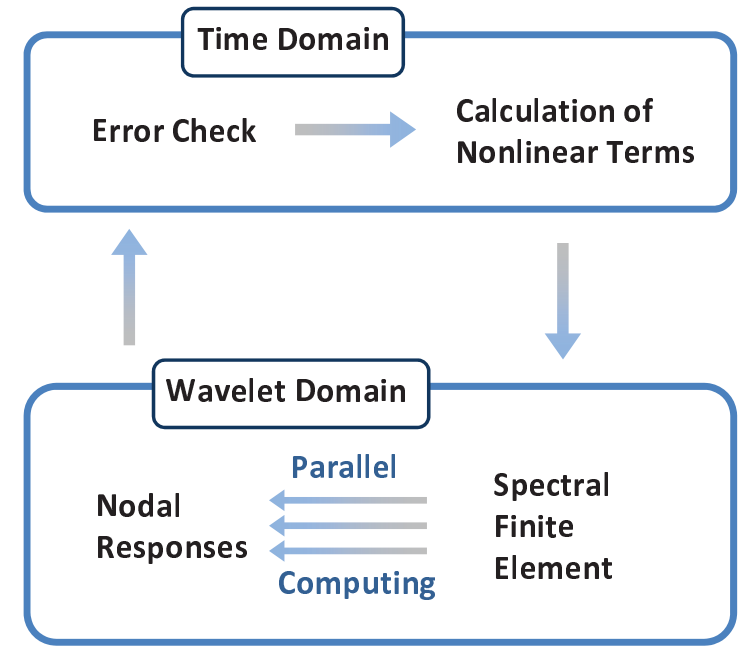

Figure 1: The flowchart for the AWT-FEM.

where

$$
F(x, t)=2 \alpha E A u^{\prime} u^{\prime \prime},
$$

the cross sectional area is represented by $A, \rho$ is the density, $u$ is the axial displacement, the prime represents a derivative with respect to the spatial coordinate $x$, and $F(x, t)$ is the nonlinear term.

By using the spectrally-uncoupled wavelet transform previously described, the governing equation of motion in the wavelet-domain is obtained as

$$
E A \hat{u}_{j}^{\prime \prime}-\rho A \lambda_{j}^{2} \hat{u}_{j}+\hat{F}_{j}=0, \quad j=0,1, \ldots, n-1 .
$$

The expression of the spectrally-dependent shape function $\mathbf{N}_{\mathbf{R}}$ for the rod model is derived from the conservative form of Eqn. (20) by assuming separation of variables and wave propagation in both directions [20]. It is a $1 \times 2$ vector with components that are functions of $x$ and $\omega$. The angular frequency $\omega$ associated with the frequency-domain implementation in the references must be replaced by $\omega=-i \lambda_{j}$ for this wavelet-domain method. By substituting it into Eqn. (17), the dynamic stiffness matrix $\mathbf{K}$ and the equivalent nodal nonlinear force term $\mathbf{q}_{\mathbf{N}}$ become

$$
\begin{aligned}
\mathbf{K} & =\int_{0}^{L_{e}} E A \mathbf{N}_{\mathbf{R}}^{\prime T} \mathbf{N}_{\mathbf{R}}^{\prime} d x+\lambda_{j}^{2} \int_{0}^{L_{e}} \rho A \mathbf{N}_{\mathbf{R}}^{T} \mathbf{N}_{\mathbf{R}} d x, \\
\mathbf{q}_{\mathbf{N}} & =\int_{0}^{L_{e}} \mathbf{N}_{\mathbf{R}}^{T} \hat{F}_{j} d x .
\end{aligned}
$$

\section{(2) Beam:}

A beam model with a geometric nonlinearity is considered here. The strain is assumed to be small and the rotation is expected to be moderately large. The von Kármán strain [10] is defined to represent the nonlinear strain-displacement relationship. The constitutive relationship is assumed to be linear. By using Hamilton's principle, the governing geometrically nonlinear homogeneous wave equations for a beam are obtained as

$$
\begin{aligned}
E A u^{\prime \prime}-\rho A \ddot{u}+F_{R}(x, t) & =0, \\
E I w^{\prime \prime \prime \prime}+\rho A \ddot{w}-F_{B}(x, t) & =0,
\end{aligned}
$$


where

$$
\begin{aligned}
& F_{R}(x, t)=E A w^{\prime} w^{\prime \prime}, \\
& F_{B}(x, t)=E A w^{\prime \prime}\left(u^{\prime}+\frac{1}{2}\left(w^{\prime}\right)^{2}\right),
\end{aligned}
$$

the axial displacement is represented by $u, w$ is the transverse displacement, $I$ is the second moment of inertia, $F_{R}(x, t)$ is the axial nonlinear force term, and $F_{B}(x, t)$ is the transverse nonlinear force term. The definitions of $E$ and $A$ are the same as those for the rod model.

By using the spectrally-uncoupled wavelet transform developed in the first subsection, the governing equations in the wavelet-domain is obtained as

$$
\begin{aligned}
E A \hat{u}_{j}^{\prime \prime}-\rho A \lambda_{j}^{2} \hat{u}_{j}+\hat{F}_{R j}(x) & =0, \\
E I \hat{w}_{j}^{\prime \prime \prime \prime}+\rho A \lambda_{j}^{2} \hat{w}_{j}-\hat{F}_{B j}(x) & =0, \quad j=0,1, \ldots, n-1 .
\end{aligned}
$$

The expression of the spectrally-dependent transverse shape functions $\mathbf{N}_{\mathbf{B}}$ is calculated in a similar manner as the axial shape functions $\mathbf{N}_{\mathbf{R}}$ from the conservative, unexcited Euler-Bernoulli beam equation [20]. The transverse shape function $\mathbf{N}_{\mathbf{B}}$ is a $1 \times 4$ vector with components that are functions of $x$ and $\omega$. As was done for rod structures, the angular frequency $\omega$ in the references must be replaced by $\omega=-i \lambda_{j}$. By organizing Eqn. (28) and Eqn. (29) into a matrix form and performing the spectral finite element formulation, the dynamic stiffness matrix $\mathbf{K}$ becomes

$$
\mathbf{K}=\left[\begin{array}{cccccc}
K_{R 11} & 0 & 0 & K_{R 12} & 0 & 0 \\
0 & K_{B 11} & K_{B 12} & 0 & K_{B 13} & K_{B 14} \\
0 & K_{B 21} & K_{B 22} & 0 & K_{B 23} & K_{B 24} \\
K_{R 21} & 0 & 0 & K_{R 22} & 0 & 0 \\
0 & K_{B 31} & K_{B 32} & 0 & K_{B 33} & K_{B 34} \\
0 & K_{B 41} & K_{B 42} & 0 & K_{B 43} & K_{B 44}
\end{array}\right]
$$

where $K_{R i j}$ and $K_{B i j}$ are components of $\mathbf{K}_{\mathbf{R}}$ and $\mathbf{K}_{\mathbf{B}}$, respectively and these matrices are defined by

$$
\begin{aligned}
& \mathbf{K}_{\mathbf{R}}=\int_{0}^{L_{e}} E A \mathbf{N}_{\mathbf{R}}^{\prime T} \mathbf{N}_{\mathbf{R}}^{\prime} d x+\lambda_{j}^{2} \int_{0}^{L_{e}} \rho A \mathbf{N}_{\mathbf{R}}^{T} \mathbf{N}_{\mathbf{R}} d x, \\
& \mathbf{K}_{\mathbf{B}}=\int_{0}^{L_{e}} E I \mathbf{N}_{\mathbf{B}}^{\prime \prime T} \mathbf{N}_{\mathbf{B}}^{\prime \prime} d x+\lambda_{j}^{2} \int_{0}^{L_{e}} \rho A \mathbf{N}_{\mathbf{B}}^{T} \mathbf{N}_{\mathbf{B}} d x .
\end{aligned}
$$

The dynamic stiffness matrix $\mathbf{K}$ is decoupled between axial and transverse motions. The equivalent nodal nonlinear force term $\mathbf{q}_{\mathbf{N}}$ becomes

$$
\mathbf{q}_{\mathbf{N}}=\left\{\begin{array}{l}
\int_{0}^{L_{e}} N_{R 1} \hat{F}_{R j} d x \\
\int_{0}^{L_{e}} N_{B 1} \hat{F}_{B j} d x \\
\int_{0}^{L_{e}} N_{B 2} \hat{F}_{B j} d x \\
\int_{0}^{L_{e}} N_{R 2} \hat{F}_{R j} d x \\
\int_{0}^{L_{e}} N_{B 3} \hat{F}_{B j} d x \\
\int_{0}^{L_{e}} N_{B 4} \hat{F}_{B j} d x
\end{array}\right\}
$$

where the terms $N_{R i}$ and $N_{B i}$ are elements of $\mathbf{N}_{\mathbf{R}}$ and $\mathbf{N}_{\mathbf{B}}$, respectively. The general spectral nodal

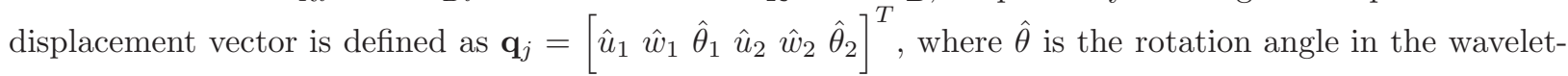
domain.

\section{(3) Plate:}


A plate model with a geometric nonlinearity is considered here. In [10], the classic and first-order plate theory with the von Kármán strain is chosen to derive the governing equation of motion. The results are three coupled nonlinear equations in the three spatial dimensions with respect to two in-plane displacements $u, v$, and one out of plane displacement $w$. The equation in the direction perpendicular to the plane can be written into the form of the von Kármán equation [30] as

$$
D \nabla^{2} \nabla^{2} w(x, y, t)+\rho h \ddot{w}(x, y, t)=F(x, y, t),
$$

where $\nabla^{2}=\partial^{2} / \partial x^{2}+\partial^{2} / \partial y^{2}$ is the Laplace operator, $h$ is the thickness of the thin plate, $w$ is the transverse displacement, $D=E h^{3} /\left(12\left(1-\nu^{2}\right)\right)$ is the flexural rigidity, and $\nu$ is the Poisson's ratio. Other parameters are defined the same as those in the rod model. The nonlinear term $F(x, y, t)$ is

$$
F(x, y, t)=N_{x x} \frac{\partial^{2} w}{\partial x^{2}}+N_{y y} \frac{\partial^{2} w}{\partial y^{2}}+2 N_{x y} \frac{\partial^{2} w}{\partial x \partial y},
$$

where $N_{x x}, N_{y y}$, and $N_{x y}$ are resultant membrane forces defined as

$$
N_{x x}=\int_{-h / 2}^{h / 2} \sigma_{x x} d z, \quad N_{y y}=\int_{-h / 2}^{h / 2} \sigma_{y y} d z, \quad N_{x y}=\int_{-h / 2}^{h / 2} \sigma_{x y} d z
$$

where the expressions of stresses $\sigma_{x x}, \sigma_{y y}$, and $\sigma_{z z}$ can be calculated from the corresponding strains.

Equation (35) indicates that the nonlinear term is a function of three unknowns $u, v$, and $w$, which correspond to displacement along the $x$-, $y$-, and $z$-axis. Two assumptions are made here to further simplify the model. First, it is assumed that the plate is only subjected to transverse loading. Second, it is assumed that there is either no axial constraints on the boundaries or the plate is large enough so that the propagating wave will not reach the boundaries within the time window. In this weak geometric nonlinear case, the inplane displacement $u$ and $v$ are very small and can be assumed to be zero in Eqn. (34) and Eqn. (35).

By using the spectrally-uncoupled wavelet transform, Eqn. (34) is transformed into the wavelet-domain as

$$
D \nabla^{2} \nabla^{2} \hat{w}_{j}(x, y)+\rho h \lambda_{j}^{2} \hat{w}_{j}(x, y)=\hat{F}_{j}(x, y) \quad j=0,1, \ldots, n-1 .
$$

The spectrally-uncoupled wavelet transform is applied again with respect to the spatial dimension $y$ and Eqn. (37) is reduced to a $1 \mathrm{D}$ problem as

$$
D\left(\frac{d^{4} \tilde{w}_{i j}}{d x^{4}}+2 \beta_{i}^{2} \frac{d^{2} \tilde{w}_{i j}}{d x^{2}}+\beta_{i}^{4} \tilde{w}_{i j}\right)+\rho h \lambda_{j}^{2} \tilde{w}_{i j}=\tilde{F}_{i j} . \quad i, j=0,1, \ldots, n-1,
$$

where $\beta_{i}$ is a pseudo spatial frequency. For more information about this spatially reduction process, refer to section 7.3 of [22] where a similar example for a linear plate model is presented.

Equation (38) is analogous to a beam equation. By setting $\tilde{F}_{i j}$ as zero, Eqn. (38) becomes a linear homogeneous wave equation. The exact solution is assumed to be $\tilde{w}_{i j}=c e^{-i k x}$ and is substituted into the linear homogeneous wave equation. Four values of the wavenumber $k\left(\beta_{i}, \lambda_{j}\right)$ are obtained through an eigenvalue analysis. The spectrally-dependent shape function $\mathbf{N}_{\mathbf{B}}$ is derived from the linearized, conservative model [20]. The wavenumber $k$ in the reference must be replaced by the new calculated values. The dynamic stiffness matrix $\mathbf{K}$ and the equivalent nodal nonlinear force term $\mathbf{q}_{\mathbf{N}}$ are derived as

$$
\begin{aligned}
\mathbf{K} & =\int_{0}^{L_{e}} D \mathbf{N}_{\mathbf{B}}^{\prime \prime T} \mathbf{N}_{\mathbf{B}}^{\prime \prime} d x-\int_{0}^{L_{e}} 2 D \beta_{i}^{2} \mathbf{N}_{\mathbf{B}}^{\prime T} \mathbf{N}_{\mathbf{B}}^{\prime} d x+\int_{0}^{L_{e}}\left(D \beta_{i}^{4}+\rho h \lambda_{j}^{2}\right) \mathbf{N}_{\mathbf{B}}^{T} \mathbf{N}_{\mathbf{B}} d x, \\
\mathbf{q}_{\mathbf{N}} & =\int_{0}^{L_{e}} \mathbf{N}_{\mathbf{B}}^{T} \tilde{F}_{i j} d x,
\end{aligned}
$$

where the explicit expressions of the integral terms are similar to the form of the expressions for the beam model. 


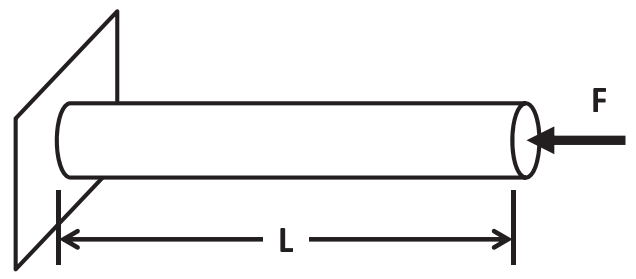

Figure 2: Diagram of a fixed-free rod.

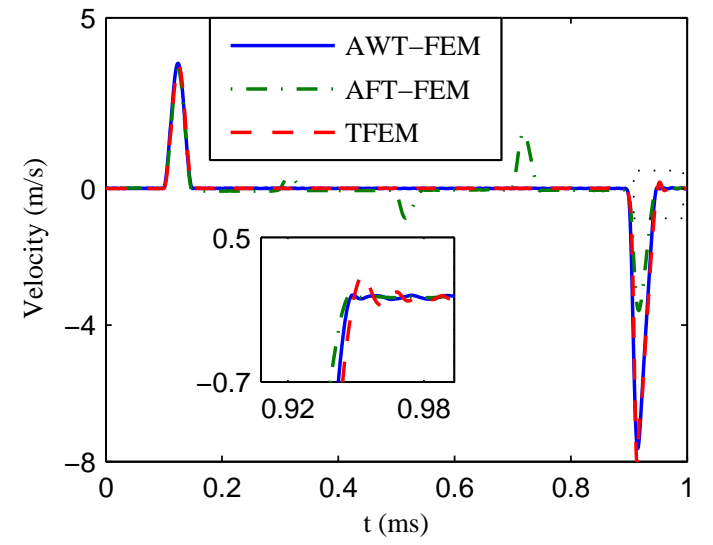

Figure 3: Velocity responses at the impacted position on the rod obtained by using AWT-FEM, AFT-FEM, and TFEM.

Table 2: System parameters of rod.

\begin{tabular}{lc}
\hline \hline Parameter & Value \\
\hline Elastic modulus, $E$ & $70 \mathrm{GPa}$ \\
Cross section, $A$ & $\pi \times 25 \mathrm{~mm} \times 25 \mathrm{~mm}$ \\
Mass density, $\rho$ & $2800 \mathrm{~kg} / \mathrm{m}^{3}$ \\
Rod length, $L$ & $2 \mathrm{~m}$ \\
Time window, $T$ & $1 \mathrm{~ms}$ \\
Impact duration, $T_{p}$ & $50 \mu \mathrm{s}$ \\
Impact amplitude, $T_{a}$ & $100 \mathrm{kN}$ \\
Sampling frequency, $f$ & $1000 \mathrm{kHz}$ \\
Nonlinear coefficient, $\alpha$ & 20 \\
\hline
\end{tabular}

Table 3: Computation time.

\begin{tabular}{ccc}
\hline \hline AWT-FEM & AFT-FEM & TFEM \\
\hline $11.9 \mathrm{~s}$ & $6.3 \mathrm{~s}$ & $25.8 \mathrm{~s}$ \\
\hline
\end{tabular}

\section{Numerical Simulations}

In the first subsection, simulations of a materially nonlinear rod are conducted by using AWT-FEM, AFT-FEM, and TFEM. The goal of this subsection is to demonstrate the advantages of AWT-FEM in fidelity and computational efficiency. Unlike beams or plates, which are strongly intrinsically dispersive, axial wave in a rod has an intact wave shape and it is easier to identify the existence of numerical errors. In the second subsection, simulations of a geometrically nonlinear beam with AWT-FEM and physically realistic boundary conditions are conducted. The influence of the interaction with the boundaries on the response is analyzed. In the third subsection, simulations of a plate model with a weak geometric nonlinearity are presented to demonstrate the adaptability of the AWT-FEM for 2D models.

\subsection{Materially Nonlinear Rod}

A diagram of a fixed-free rod subjected to an axial impact load at one end is shown in Fig. 2. The parameters of the system are presented in Table 2. The rod is modeled with a nonlinear constitutive relationship as defined in Eqn. (19) with a coefficient $\alpha=20$.

Simulation results for the materially nonlinear rod model in Eqn. (20) with AWT-FEM, AFT-FEM, and TFEM are shown in Fig. 3. The Daubechies wavelet with an order of $N=14$ is used for the spectrallyuncoupled wavelet transform and can yield sufficient smoothness in the responses. Fifty spectral rod elements are used for the AWT-FEM and the AFT-FEM models and 350 linear rod elements are used for TFEM. For systems with physically realistic boundaries, additional damping combined with a longer time-window is required for the AFT-FEM to avoid wrap-around errors. However, convergence issues can occur for the 


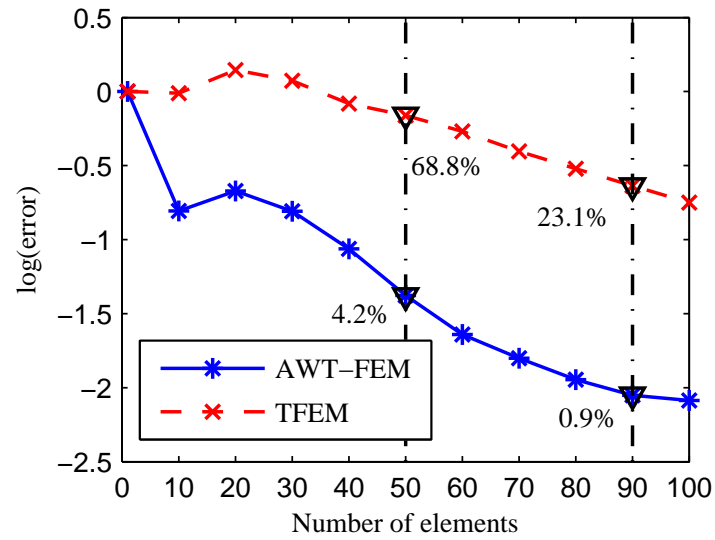

Figure 4: Comparison of convergence between the AWT-FEM and the nonlinear TFEM when the impact amplitude $T_{a}=$ $100 \mathrm{kN}$ and the nonlinear coefficient $\alpha=20$.

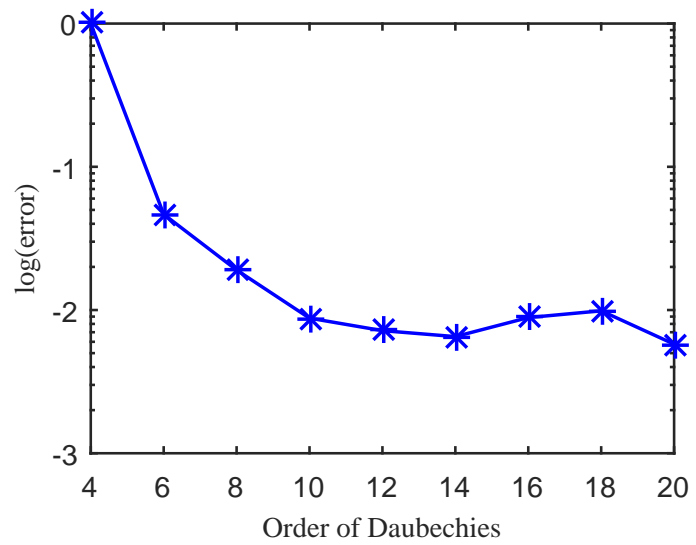

Figure 5: Convergence of the order of Daubechies wavelet.

AFT-FEM iterative procedure with a long time-window. In the simulation, the AFT-FEM is not able to produce a reliable physical response. In order to obtain a converged result for comparison with other methods, a large damping factor $\eta=1 \times 10^{4}$ combined with the original time window is used. The damping factor is multiplied by $\dot{u}$ and added into Eqn. (20).

An impulse in the velocity is created by the impact load at $0.1 \mathrm{~ms}$. The wave is reflected by the fixed end and travels back to the impacted position at $0.9 \mathrm{~ms}$. In the reflected wave, the slope of the wave shape on the left hand side becomes steeper and the slope on the right hand side becomes comparatively shallower. Overall, the wave shape is observed to lean to the left-hand side. This distortion of wave shape caused by the nonlinearity is captured by all three methods. Due to the additional damping, the amplitude of the response simulated by using AFT-FEM is severely reduced. Wrap-around errors also occurs in the time-history result in additional oscillations. These results demonstrate that the AFT-FEM cannot work for nonlinear structures without the use of non-reflecting boundaries. In the comparison, a physical boundary is presented and the same time-window is adopted for all three numerical methods. The TFEM can produce a response with adequate resolution at the expense of a refined mesh (350 elements). However, in the insert of Fig. 3, the reflected wave is affected by spurious oscillations late in the time series resulting from erroneously introduced numerical dispersion. For an impact load with shorter duration and higher frequency content, this error can be further magnified and completely distort the response [15]. On the contrary, AWT-FEM only uses 50 elements to produce an intact wave response and capture the nonlinear distortion without loss of quality.

The computation times for the three methods are listed in Table 3. A Dell desktop with a Intel Core Quad CPU is used for all simulations. The parallel computing toolbox in MATLAB is used to execute the calculation over a range of pseudo-frequency in parallel. With the use of parallel computing, the AWTFEM only uses $11.9 \mathrm{~s}$ with 50 elements to obtain an accurate response while the TFEM needs $25.8 \mathrm{~s}$ with 350 elements. The AFT-FEM cannot produce a correct response for these conditions. If a fast numerical technique similar to FFT can be applied to modify the wavelet transform procedure, the computational performance of the AWT-FEM will be further improved.

A convergence study of the AWT-FEM and the TFEM is conducted. The impact condition described in Table 2 is adopted. Simulations start with 1 elements and incrementally refine the mesh by adding 10 elements. The error is defined as the absolute value of the relative difference between the current simulation result of the velocity response $V_{\text {cur }}$ and the reference in the previous state $V_{\text {pre }}$ at the impacted position, as defined in Eqn. (41). The state being considered can be the number of elements or order of Daubechies wavelet depending on the convergence study. The convergence studies of the two methods are performed separately for each of them to obtain their own convergence information. They do not share a common 


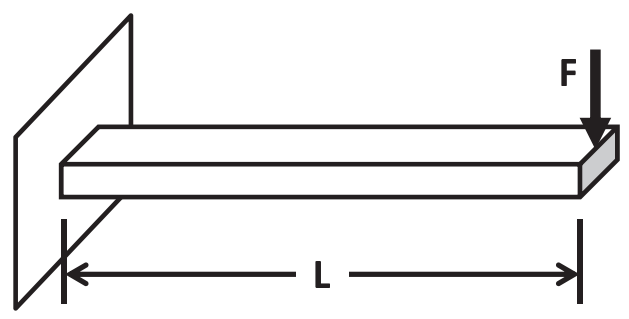

Figure 6: Diagram of a cantilevered beam.

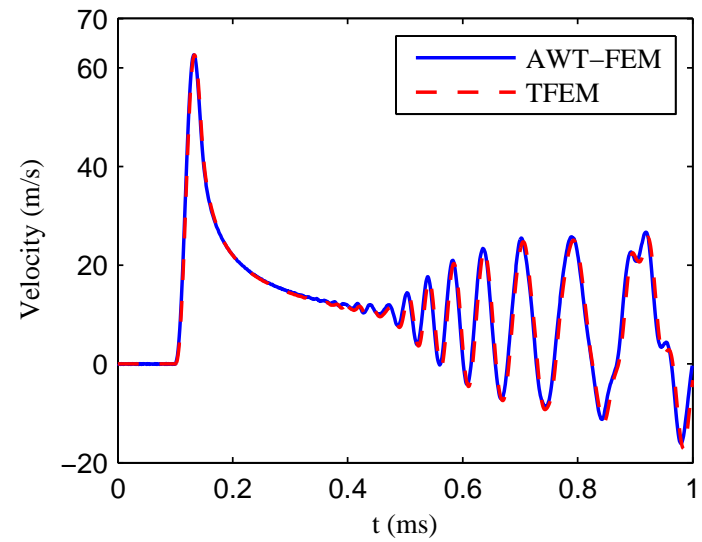

Figure 7: Velocity responses at the impacted position on the beam obtained by using AWT-FEM and TFEM.

converged solution. As demonstrated above, TFEM introduces numerical dispersion into the response.

$$
\text { Error }=\frac{\sum_{i=1}^{n}\left\|V_{\text {cur }}\left(t_{i}\right)-V_{\text {pre }}\left(t_{i}\right) V\right\|}{\sum_{i=1}^{n}\left\|V_{\text {cur }}\left(t_{i}\right) V\right\|} .
$$

As show in Fig. 4, the convergence performance of the AWT-FEM is significantly better than the TFEM. With 50 elements, the AWT-FEM has a $4.2 \%$ error level while the error of TFEM is $68.8 \%$. By increasing the number of elements to 90 , the AWT-FEM can reach a $0.9 \%$ error level while the TFEM still has an error of $23.1 \%$.

A convergence study of the AWT-FEM with respect to the order of Daubechies wavelet is also conducted with the same error measure defined in Eqn. (41). As shown in Fig. 5, the order of Daubechies wavelet from 4 to 20 with an increment of 2 is considered. When the order is greater than 8 , the error level is below $1 \%$.

\subsection{Geometrically Nonlinear Beam}

Table 4: System parameters of a beam

\begin{tabular}{ll}
\hline \hline Parameter & Value \\
\hline Elastic modulus, $E$ & $70 \mathrm{GPa}$ \\
Cross section, $A$ & $25 \mathrm{~mm} \times 25 \mathrm{~mm}$ \\
Moment of inertia, $I$ & $3.26 \times 10^{-8} \mathrm{~m}^{4}$ \\
Mass density, $\rho$ & $2800 \mathrm{~kg} / \mathrm{m}^{3}$ \\
Beam length, $L$ & $1 \mathrm{~m}$ \\
Time window, $T$ & $1 \mathrm{~ms}$ \\
Impact duration, $T_{p}$ & $50 \mu \mathrm{s}$ \\
Impact amplitude, $T_{a}$ & $100 \mathrm{kN}$ \\
Sampling frequency, $f$ & $1000 \mathrm{kHz}$ \\
\hline
\end{tabular}

A diagram of a cantilevered beam with clamped-free boundaries, as shown in Fig. 6, is studied. A point impact load with an amplitude $150 \mathrm{kN}$ is applied at the free end. The parameters of the system are presented in Table 4. Simulations for the geometrically nonlinear beam model in Eqn. (24) and Eqn. (25) with the clamped-free boundaries is conducted by using AWT-FEM. A Daubechies wavelet with an order of $N=14$ 


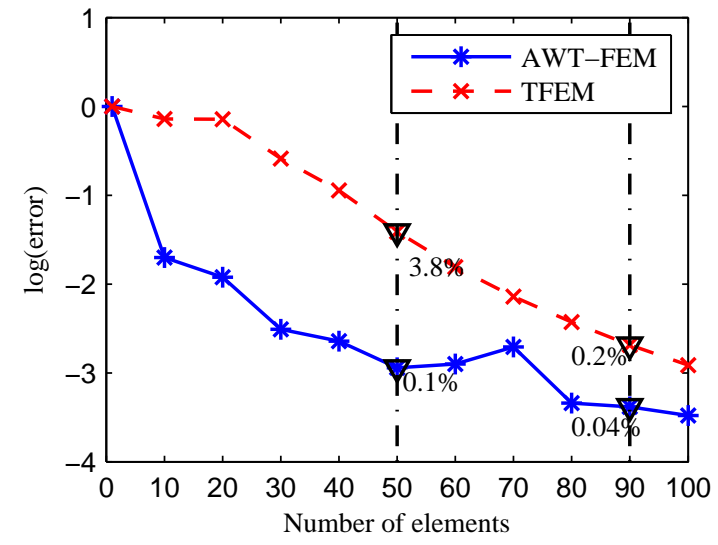

Figure 8: Comparison of convergence between the AWT-FEM and the nonlinear TFEM when the impact amplitude $T_{a}=$ $100 \mathrm{kN}$

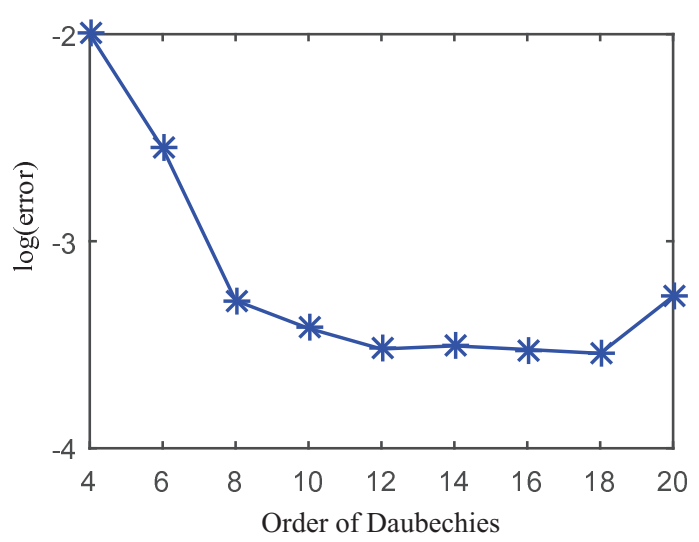

Figure 9: Convergence of the order of Daubechies wavelet.

is used for the spectrally-uncoupled wavelet transform and can yield sufficient smoothness in the response. Fifty elements are used in the spectral element formulation to provide satisfactory accuracy and sufficient resolution.

A comparison of the velocity response at the impacted position obtained by using the AWT-FEM and a nonlinear TFEM is shown in Fig. 7. The AWT-FEM uses 50 elements and 100 elements are used for TFEM. As reported in previous research, the geometric nonlinearities have limited influence on transverse wave propagation in beams in the intermediate strain regime [33, 34] as compared to the nonlinear dispersion in the rod model. Since the beam is a strong intrinsically nonlinear system, the influence of the nonlinear dispersion introduced by geometric nonlinearity on the intrinsic dispersion relationship of the beam in the intermediate strain regime is negligible. The nonlinear responses obtained by using the two methods are close to each other. The difference in the later part of the response, where the reflected signal interacts with the forward traveling waves, may be attributed to the extra dispersion introduced by the TFEM.

A convergence study of the AWT-FEM and the TFEM for the beam model is conducted. The same impact conditions described in Table 4 are adopted. Simulations start with 1 and 10 elements and incrementally refine the mesh by adding 10 elements. The error measure defined in Eqn. (41) is adopted here. The error level of the AWT-FEM is two orders of magnitude smaller than the TFEM. With 50 elements, the AWT-FEM has only $0.1 \%$ error while the TFEM has an error of $3.8 \%$. By increasing the number of elements to 90 , the error of the AWT-FEM reduces to $0.04 \%$ while the TFEM is $0.2 \%$.

A convergence study of the AWT-FEM with respect to the order of the Daubechies wavelet is also conducted with the same error measure defined in Eqn. (41) for the beam model. As shown in Fig. 9, the order of the Daubechies wavelet is studied from 4 to 20 with an increment of 2 . When the order is greater than 6 , the error level is below $0.01 \%$.

\subsection{Geometrically Nonlinear Plate}

A diagram of a cantilevered plate with clamped-free boundaries along the $x$-dimension and free-free boundaries along the $y$-dimension is shown in Fig. 10. A point impact load is applied at the middle point of edge $B C\left(L_{y}\right)$. Other general distributed loads could also be chosen. The parameters of the system are presented in Table 5. Along the $y$-dimension, the free-free boundaries place no constraints on the axial motion. Along the $x$-dimension, the length is chosen to be $L_{x}=1 \mathrm{~m}$, the same as was used for the beam model in previous subsection. The width is chosen to be $L_{y}=0.1 \mathrm{~m}$ to approximate a narrow $2 \mathrm{D}$ cantilever beam. The two models are equivalent to each other and the narrow beam is used so that the responses may be compared. 


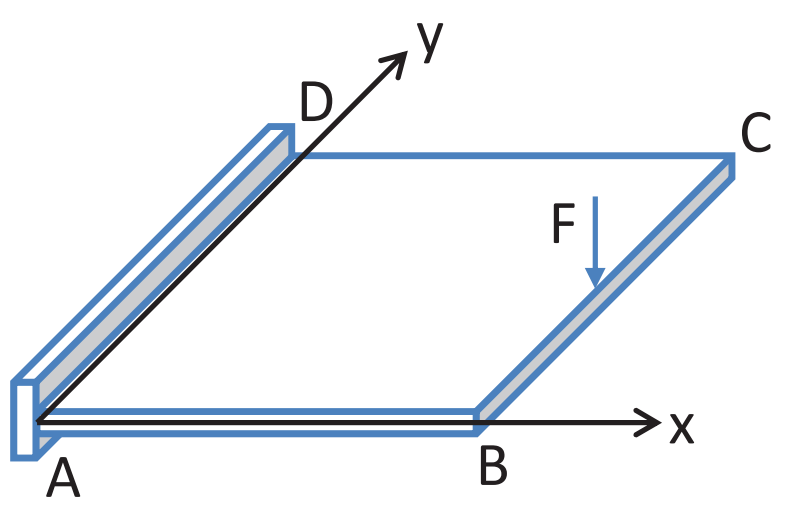

Figure 10: Diagram of a cantilevered plate.

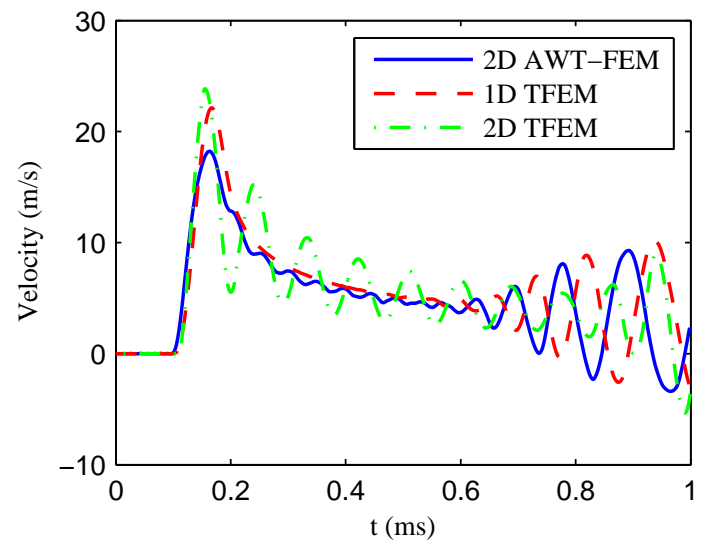

Figure 11: Velocity responses at the impacted position on the beam obtained using AWT-FEM, 1D TFEM, and 2D TFEM.

Table 5: System parameters of a plate

\begin{tabular}{ll}
\hline \hline Parameter & Value \\
\hline Elastic modulus, $E$ & $70 \mathrm{GPa}$ \\
Density, $\rho$ & $2800 \mathrm{~kg} / \mathrm{m}^{3}$ \\
Length $1, L_{x}$ & $1 \mathrm{~m}$ \\
Length $2, L_{y}$ & $0.1 \mathrm{~m}$ \\
Thickness, $h$ & $25 \mathrm{~mm}$ \\
Poisson's ratio, $\nu$ & 0.33 \\
Time window, $T$ & $1 \mathrm{~ms}$ \\
Impact amplitude, $F_{m}$ & $100 \mathrm{kN}$ \\
Impact duration, $T_{p}$ & $100 \mu \mathrm{s}$ \\
Sampling frequency, $f$ & $500 \mathrm{kHz}$ \\
\hline
\end{tabular}

Simulations for a plate model with a weak geometric nonlinearity based on Eqn. (34) in the intermediate strain value regime is conducted by using AWT-FEM. A Daubechies wavelet with an order of $N=22$ is used for the wavelet transform with respect to time. A Daubechies wavelet with an order of $N=16$ with a sampling rate $\Delta y=0.04 \mathrm{~m}$ is used for the wavelet transform with respect to the spatial coordinate $y$. These wavelets are able to provide sufficient smoothness in the response. Twenty elements are used to discretize the structure in both the $x$ - and $y$-dimensions.

The comparison of the velocity responses at the impacted position obtained by using the AWT-FEM, 1D TFEM, and 2D TFEM are shown in Fig. 11. The 1D TFEM with 100 elements is applied onto a 1D beam model with the same length as in the previous subsection. Since the width of the plate is narrow, the response of the AWT-FEM has a similar trend as the response of the 1D TFEM. There are three major differences between the responses of the two models. First, the width of the plate makes it stiffer than the corresponding 1D beam model, which results in a smaller amplitude of the response in the AWT-FEM. Second, after the initial pulse of the the AWT-FEM response, small amplitude oscillations occur due to interactions with the reflected waves from both sides while the response in the 1D TFEM is smooth. Third, the later portions of the responses in the two methods when the reflected waves return are different. This may be related to the effect of the sides of the plate and the influence of the different stiffness conditions on the wave speed. A 2D TFEM model with a $50 \times 20$ elements using ANSYS is also adopted here to produce the wave propagation process. As shown by the dashed-doted green line, there are differences between the two responses. As previously observed, the TFEM simulations are unable to converge to the AWT-FEM results, even when 
the mesh resolution is significantly increased. For the condition of extreme impact loading, the 2D TFEM with a reasonable fine mesh using a sequential time integrator cannot produce a convergent result. It is also worth noting that by further increasing the impact load and leading the strain into a strong value regime, all three methods will encounter convergence issue.

\section{Conclusions and Future Work}

The alternating wavelet-time finite element method (AWT-FEM) proposed in this paper has shown the potential to meet the three criteria identified in the introduction section: (1) It has the potential to produce high fidelity results through the use of the spectral finite element method and this could be used to accurately capture nonlinear behavior without distortions from numerical errors. (2) The parallel computing framework can improve the computational efficiency. (3) AWT-FEM can be applied to study wave propagation in structures with a general nonlinearity and different physical boundary conditions.

The direct iteration algorithm adopted in this study limits the convergence performance of the AWTFEM. For 1D beam and 2D plate with geometric nonlinearity, the AWT-FEM can only obtain a converged response within an intermediate strain value regime $\left(10^{-6}\right.$ to $\left.10^{-3}\right)$ where the influence of the geometric nonlinearity is limited. As the strain value becomes larger, the algorithm may stagnate over iterations or eventually become non-convergent. A modified Newton-Raphson iterative method will be used to replace the direct iteration algorithm in order to improve the convergence performance of the AWT-FEM and make it applicable for large deformation problems.

AWT-FEM has shown the potential to become an efficient tool for studying nonlinear wave propagation in one-dimensional structural components. Its applications in areas such as force identifications in aircraft, high accuracy control and analysis of parallel mechanisms, and vibration and wave propagation of drill strings will be explored in future work.

\section{Acknowledgment}

The support of this work from the Air Force Office of Scientific Research under Grant FA9550-11-1-0108 is gratefully acknowledged.

\section{Appendix A.}

\section{A.1 Connection Coefficients}

For a nonlinear 1D rod model, as shown in Eqn. (20), by substituting Eqn. (5) and Eqn. (7) into Eqn. (20), multiplying by the scaling function $\varphi(\tau-j)$, and integrating with respect to $\tau$ from $-\infty$ to $+\infty$, the equation of motion becomes

$$
\begin{aligned}
E A \sum_{k} u_{k}^{\prime \prime} \int_{-\infty}^{\infty} \varphi(\tau-k) \varphi(\tau-j) d \tau= & \frac{\rho A}{\Delta t^{2}} \sum_{k} u_{k} \int_{-\infty}^{\infty} \ddot{\varphi}(\tau-k) \varphi(\tau-j) d \tau- \\
& \sum_{k} F_{R k} \int_{-\infty}^{\infty} \varphi(\tau-k) \varphi(\tau-j) d \tau .
\end{aligned}
$$

By utilizing the orthogonality of the scaling function, the above equation becomes

$$
E A u_{j}^{\prime \prime}=\sum_{k=j-N+2}^{j+N-2} \frac{\rho A}{\Delta t^{2}} \Omega_{j-k}^{2} u_{k}-F_{R j}, \quad j=0,1, \cdots, n-1
$$

where $n$ is the length of the sample, $N$ is the order of the Daubechies wavelet, and $\Omega_{j-k}^{2}$ is the connection coefficients defined as in Eqn. (11). The inclusion of both the $j$ and $k$ subscript results from the orthogonality conditions where the $j$ coefficients in Eqn. (A.2) result from conditions where $k=j$ and the remaining $k$ coefficient is related to the $j$ coefficients by way of the connection coefficients $\Omega_{j-k}^{2}$. 
An iterative algorithm [27] is adopted to calculate the values of integrals in the above equations. For a given order of the Daubechies wavelet $N$, the values of all $\Omega_{j-k}^{1}$ and $\Omega_{j-k}^{2}$ can be calculated and stored for future use.

The matrix form of Eqn. (A.2) is

$$
E A\left\{u_{j}\right\}^{\prime \prime}=\rho A \Omega^{2}\left\{u_{k}\right\}-\left\{F_{R j}\right\},
$$

where the connection coefficient matrix $\Omega^{2}$ is composed as

$$
\Omega^{2}=\frac{1}{\Delta t^{2}}\left[\begin{array}{cccccccc}
\Omega_{N-2}^{2} & \Omega_{N-3}^{2} & \ldots & \Omega_{-N+3}^{2} & \Omega_{-N+2}^{2} & & 0 & \\
& \Omega_{N-2}^{2} & \Omega_{N-3}^{2} & \ldots & \Omega_{-N+3}^{2} & \Omega_{-N+2}^{2} & & \\
& & \ddots & \ddots & \ddots & \ddots & \ddots & \\
0 & & & \Omega_{N-2}^{2} & \Omega_{N-3}^{2} & \ldots & \Omega_{-N+3}^{2} & \Omega_{-N+2}^{2}
\end{array}\right],
$$

the $n \times 1$ vector of $\left\{u_{j}\right\}$ and $(2 N-4+n) \times 1$ vector of $\left\{u_{k}\right\}$ are defined as

$$
\begin{aligned}
& \left\{u_{j}\right\}=\left[u_{0}, u_{1}, \cdots, u_{n-2}, u_{n-1}\right]^{T}, \\
& \left\{u_{k}\right\}=\left[u_{-N+2}, u_{-N+3}, \cdots, u_{-1}, u_{0}, u_{1}, \cdots, u_{n-1}, u_{n}, \cdots, u_{n+N-3}\right]^{T} .
\end{aligned}
$$

The connection coefficient matrix $\boldsymbol{\Omega}^{1}$ has the same form as defined above (replacing superscript 2 with 1 and using Eqn. (10) instead of Eqn. (11)). The matrix form is a commonly used convention to relate two vectors with different dimensions. The zero values and non-zero values indicate which elements in $\left\{u_{k}\right\}$ contribute to the value of elements in $\left\{u_{j}\right\}$.

The values of $u_{0}, u_{1}, \cdots, u_{n-1}$ are directly related to the corresponding sample points in the signal. The values of $u_{-N+2}, \cdots, u_{-1}$ and $u_{n}, \cdots, u_{n+N-3}$ are unknowns and need to be calculated from $u_{0}, u_{1}, \cdots, u_{n-1}$ by using an extrapolation technique introduced in the following subsection.

\section{A.2 Non-periodic Boundary}

A wavelet extrapolation method is adopted here to construct a non-periodic time boundary. The left-hand side (LHS) of the time series of data $\left\{u_{k}\right\}$ defined in Eqn. (A.6) is an initial value problem. The unknown coefficients $u_{-N+2}, u_{-N+3}, \cdots, u_{-2}, u_{-1}$ are extrapolated from the initial values. On the right-hand-side (RHS), the values of unknowns $u_{n}, \cdots, u_{n+N-3}$ are extrapolated from the values of $u_{n-1-p+1}, \cdots, u_{n-1}$, where $p=N / 2$.

The extrapolation for the RHS for a 1D model is taken as an example here. The moments of the scaling function [32] are defined as

$$
\mu_{j}^{l}=\int_{-\infty}^{\infty} \tau^{l} \varphi(\tau-j) d \tau
$$

Define a matrix $\mathbf{D}$ as

$$
\mathbf{D}=\left[\begin{array}{cccc}
\mu_{-M}^{0} & \mu_{-M}^{1} & \cdots & \mu_{-M}^{p-1} \\
\mu_{-M+1}^{0} & \mu_{-M+1}^{1} & \cdots & \mu_{-M+1}^{p-1} \\
\vdots & \vdots & \vdots & \vdots \\
\mu_{-1}^{0} & \mu_{-1}^{1} & \cdots & \mu_{-1}^{p-1}
\end{array}\right]
$$

where $M=N$, and define a matrix $\mathbf{F}$ as

$$
\mathbf{F}=\left[\begin{array}{cccc}
\mu_{0}^{0} & \mu_{0}^{1} & \cdots & \mu_{0}^{p-1} \\
\mu_{1}^{0} & \mu_{1}^{1} & \cdots & \mu_{1}^{p-1} \\
\vdots & \vdots & \vdots & \vdots \\
\mu_{N-3}^{0} & \mu_{N-3}^{1} & \cdots & \mu_{N-3}^{p-1}
\end{array}\right]
$$


The extrapolation relation is established as

$$
\left\{\begin{array}{c}
u_{n} \\
u_{n+1} \\
\vdots \\
u_{n+N-3}
\end{array}\right\}=\mathbf{F} \times\left(\left(D^{T} D\right)^{-1} D^{T}\right)\left\{\begin{array}{c}
u_{n-M} \\
u_{n-M+1} \\
\vdots \\
u_{n-1}
\end{array}\right\} .
$$

By substituting the above result into Eqn. (A.3) and rearranging the matrix, the unknown coefficients $u_{-N+2}, u_{-N+3}, \cdots, u_{-2}, u_{-1}$ and $u_{n}, \cdots, u_{n+N-3}$ are eliminated, and the connection coefficient matrix $\boldsymbol{\Omega}^{2}$ becomes $\Lambda^{2}$, which is the version with non-periodic time boundaries. The matrix form in Eqn. (A.3) becomes

$$
E A\left\{u_{j}\right\}^{\prime \prime}=\rho A \Lambda^{2}\left\{u_{j}\right\}-\left\{F_{R j}\right\} .
$$

\section{A.3 Decoupling}

In Eqn. (A.11), the connection coefficient matrix $\Lambda$ is a $n \times n$ matrix. An eigenvalue analysis is performed to diagonalized the matrix and decouple the ODEs. The eigenvalues of $\boldsymbol{\Lambda}^{2}$ are $\lambda_{j}, j=0,1, \cdots, n-1$. The $n \times n$ eigenvector matrix of $\boldsymbol{\Lambda}^{2}$ is redefined as $\boldsymbol{\Phi}$. The decoupled form of Eqn. (A.11) is obtained as

$$
E A \hat{u}_{j}^{\prime \prime}=\rho A \lambda_{j}^{2} \hat{u}_{j}-F_{R j},
$$

where

$$
\left\{\hat{u}_{j}\right\}=\boldsymbol{\Phi}^{-1}\left\{u_{j}\right\}
$$

\section{References}

[1] P. Taylor, C. Ford, Simulation of blast-induced early-time intracranial wave physics leading to traumatic brain injury, Journal of Biomechanical Engineering 131 (6) (2009) 61007-61018.

[2] M. Meo, G. Zumpano, Nonlinear elastic wave spectroscopy identification of impact damage on a sandwich plate, Composite structures 71 (3-4) (2005) 469-474.

[3] P. A. Johnson, X. Jia, Nonlinear dynamics, granular media and dynamic earthquake triggering, Nature 437 (7060) (2005) 871-874.

[4] P. Ghaderi, A. J. Dick, J. R. Foley, G. Falbo, Spectral domain force identification of impulsive loading in beam structures, in: Topics in Nonlinear Dynamics, Volume 3, Springer, 2012, pp. 157-165.

[5] Y. Khulief, H. Al-Naser, Finite element dynamic analysis of drillstrings, Finite elements in analysis and design 41 (13) (2005) 1270-1288.

[6] J. M. Carcione, F. Poletto, G. Pinna, Simulation of flexural waves in drill pipes including the effects of the gravitational field: Flexural waves and effects of gravity, Wave Motion 50 (2) (2012) 310-325.

[7] G. Piras, W. Cleghorn, J. Mills, Dynamic finite-element analysis of a planar high-speed, high-precision parallel manipulator with flexible links, Mechanism and Machine Theory 40 (7) (2005) 849-862.

[8] J. Doyle, Wave Propagation in Structures, Springer, Berlin, Germany, 1997.

[9] A. Nayfeh, D. Mook, Nonlinear oscillations, John Wiley, New York, USA, 1995.

[10] J. Reddy, An introduction to nonlinear finite element analysis, Oxford University Press, Oxford, UK, 2004.

[11] S. Ham, K. Bathe, A finite element method enriched for wave propagation problems, Computers and structures 94-95 (2012) $1-12$.

[12] J. Doyle, A spectrally formulated finite element for longitudinal wave propagation, International Journal of Analytical and Experimental Modal Analysis 3 (1988) 1-5.

[13] M. Mitra, S. Gopalakrishnan, Spectrally formulated wavelet finite element for wave propagation and impact force identification in connected 1-d waveguides, International journal of solids and structures 42 (16) (2005) 4695-4721.

[14] Y. Liu, P. Ghaderi, A. Dick, High fidelity methods for modeling nonlinear wave propagation in one-dimensional waveguides, in: Proceedings of the ASME 2012 International Mechanical Engineering Congress and Exposition, Houston, Texas, 2012.

[15] A. Idesman, A new high-order accurate continuous galerkin method for linear elastodynamics problems, Computational Mechanics 40 (2) (2007) 261-279.

[16] P. Kudela, M. Krawczuk, W. Ostachowicz, Wave propagation modelling in 1d structures using spectral finite elements, Journal of Sound and Vibration 300 (1) (2007) 88-100.

[17] A. T. Patera, A spectral element method for fluid dynamics: laminar flow in a channel expansion, Journal of Computational Physics 54 (3) (1984) 468-488.

[18] A. Ramabathiran, S. Gopalakrishnan, Time and frequency domain finite element models for axial wave analysis in hyperelastic rods, Mechanics of advanced materials and structures 19 (1-3) (2012) 79-99. 
[19] S. Gopalakrishnan, A. Chakraborty, D. R. Mahapatra, Spectral Finite Element Method: Wave Propagation, Diagnostics and Control in Anisotropic and Inhomogenous Structures, Springer, London, UK, 2008.

[20] U. Lee, Spectral element method in structural dynamics, Wiley, Chichester, UK, 2009.

[21] J. R. Williams, K. Amaratunga, A discrete wavelet transform without edge effects using wavelet extrapolation, Journal of Fourier Analysis and Applications 3 (4) (1997) 435-449.

[22] S. Gopalakrishnan, M. Mitra, Wavelet methods for dynamical problems: With application to metallic, composite, and nano-composite structures, CRC Press, New York, USA, 2010.

[23] L. Pahlavan, C. Kassapoglou, A. Suiker, Z. Gürdal, A 2d wavelet-based spectral finite element method for elastic wave propagation, Philosophical Magazine 92 (28-30) (2012) 3699-3722.

[24] L. Pahlavan, C. Kassapoglou, Z. Gürdal, Spectral formulation of finite element methods using daubechies compactlysupported wavelets for elastic wave propagation simulation, Wave Motion 50 (3) (2012) 558-578.

[25] T. Cameron, J. Griffin, An alternating frequency/time domain method for calculating the steady-state response of nonlinear dynamic systems, Journal of applied mechanics 56 (1) (1989) 149-154.

[26] U. Lee, I. Jang, Nonlinear spectral element model for the blood flow in human arteries, in: Proceedings of the 2011 International Symposium of Computational Models for Life Sciences, Toyama City, Japan, 2011, pp. 136-145.

[27] G. Beylkin, On the representation of operators in bases of compactly supported wavelets, SIAM Journal on Numerical Analysis 29 (6) (1992) 1716-1740.

[28] C. S. Burrus, R. A. Gopinath, H. Guo, J. E. Odegard, I. W. Selesnick, Introduction to wavelets and wavelet transforms: a primer, Vol. 23, Prentice hall, New Jersey, USA, 1998.

[29] J. Li, Y. Zhang, Exact travelling wave solutions in a nonlinear elastic rod equation, Applied Mathematics and Computation 202 (2) (2008) 504-510.

[30] T. von Kármán, Fesigkeitsprobleme in maschinenbau, Encycl de Math Wiss 4 (1910) 348-351.

[31] Y. Liu, A. Dick, Numerical analysis of transient wave propagation in nonlinear one-dimensional waveguides by using the spectral finite element method, ASME Journal of Computational Dynamics 10 (5) (2014).

[32] I. Daubechies, Ten lectures on wavelets, SIAM, 29 (6) (1992).

[33] H. Abedinnasab, I. Hussein, Wave dispersion under finite deformation, Wave Motion 50 (3) (2012) 374-388.

[34] H. Xu, M. Day, H, Minster, Hysteresis and two-dimensional nonlinear wave propagation in berea sandstone, Journal of geophysical research 105 (B3) (2000) 6163-6175.

[35] T. Hughes, G. Hulbert, Space-time finite element methods for elastodynamics: formulations and error estimates, Computer methods in applied mechanics and engineering 66 (3) (2000) 339-363. 
Table 1: Comparison of different methods.

\begin{tabular}{|c|c|c|c|c|c|}
\hline Methods & High Fidelity & Linear & Nonlinear & Boundary & $\overline{\text { Parallel }}$ \\
\hline TFEM [11] & $\bar{x}$ & $\sqrt{ }$ & $\sqrt{ }$ & $\sqrt{ }$ & $x$ \\
\hline MTFEM [12] & $\sqrt{ }$ & $\checkmark$ & $\checkmark$ & $\sqrt{ }$ & $x$ \\
\hline SFEM [13] & $\sqrt{ }$ & $\sqrt{ }$ & $x$ & - & $\sqrt{ }$ \\
\hline WSFEM [14] & $\sqrt{ }$ & $\sqrt{ }$ & $x$ & $\checkmark$ & $\checkmark$ \\
\hline AFT-FEM [15] & $\sqrt{ }$ & $\sqrt{ }$ & $\sqrt{ }$ & - & $\sqrt{ }$ \\
\hline AWT-FEM & $\checkmark$ & $\sqrt{ }$ & $\checkmark$ & $\sqrt{ }$ & $\sqrt{ }$ \\
\hline
\end{tabular}


Table 2: System parameters of a rod

\section{Parameter Value}

Elastic modulus, $E \quad 70 \mathrm{GPa}$

Cross section, $A$

$\pi \times 25 \mathrm{~mm} \times 25 \mathrm{~mm}$

Mass density, $\rho$

$2800 \mathrm{~kg} / \mathrm{m}^{3}$

Rod length, $L$

$2 \mathrm{~m}$

Time window, $T$

$1 \mathrm{~ms}$

Impact duration, $T_{p}$

$50 \mu \mathrm{s}$

Impact amplitude, $T_{a}$

$100 \mathrm{kN}$

Sampling frequency, $f$

$1000 \mathrm{kHz}$

Nonlinear coefficient, $\alpha \quad 20$ 


\section{Table 3: Computation time}

$$
\begin{array}{lll}
\text { AWT-FEM } & \text { AFT-FEM } & \text { TFEM } \\
\hline 11.9 \mathrm{~s} & 6.3 \mathrm{~s} & 25.8 \mathrm{~s}
\end{array}
$$


Table 4: System parameters of a beam

\section{Parameter Value}

Elastic modulus, $E \quad 70 \mathrm{GPa}$

Cross section, $A$

$25 \mathrm{~mm} \times 25 \mathrm{~mm}$

Mass density, $\rho$

$2800 \mathrm{~kg} / \mathrm{m}^{3}$

Beam length, $L$

$1 \mathrm{~m}$

Time window, $T$

$1 \mathrm{~ms}$

Impact duration, $T_{p}$

$50 \mu \mathrm{s}$

Sampling frequency, $f \quad 1000 \mathrm{kHz}$ 
Table 5: System parameters of a plate

\section{Parameter Value}

Elastic modulus, $E \quad 70 \mathrm{GPa}$

Density, $\rho$

$2800 \mathrm{~kg} / \mathrm{m}^{3}$

Length $1, L_{x}$

$4 \mathrm{~m}$

Length 2, $L_{y}$

$4 \mathrm{~m}$

Thickness, $h$

$25 \mathrm{~mm}$

Poisson's ratio, $\nu$

0.33

Time window, $T$

$1 \mathrm{~ms}$

Impact amplitude, $F_{m}$

$5000 \mathrm{kN}$

Impact duration, $T_{p}$

$50 \mu \mathrm{s}$

Sampling frequency, $f$

$500 \mathrm{kHz}$ 